\title{
Effet de différentes matières grasses sur les caractéristiques du comportement alimentaire et mérycique chez la vache laitière
}

\author{
B Matatu 1, E Teller 1, M Vanbelle 1, W Van Hofstraeten 2, G Van Aelten 2 \\ 1 Laboratoire de Biochimie de la nutrition, UCL, place Croix-du-Sud, 2/8, 1348 Louvain-la-Neuve; \\ 2 AVEVE, Mierdsedijk 116, 2382 Poppel, Belgique
}

\begin{abstract}
Summary - Effect of different fat supplements on chewing behaviour by lactating dairy cows. Different fat sources were tested with regard to their effect on rate of intake and ruminating behaviour in lactating dairy cows. Soybean oil increased unitary ruminating time, whereas no effect was observed with whole roasted soybeans. Coconut + palm cake also increased the time the animals spent chewing per $\mathrm{kg} \mathrm{DM}$ intake.
\end{abstract}

L'incorporation de matières grasses aux rations peut diminuer la dégradation des constituants alimentaires dans le rumen et, de cette manière, réduire les quantités ingérées (Anderson et al, 1979). Elle peut également diminuer l'appétibilité des aliments concentrés, se traduisant par une diminution de la vitesse d'ingestion (Doreau, 1984). L'objectif du présent travail est d'étudier le comportement almentaire et mérycique de vaches laitières recevant, dans leur aliment concentré, différentes matières grasses animales ou végétales couramment utilisées.

Matériel et méthodes - Deux expériences ont été réalisées, avec 4 vaches FrisonneHolstein d'un poids vif moyen de $570 \mathrm{~kg}$ entre le troisième et le septième mois de lactation. Quatre rations, constituées d'ensilage de maïs (35\% de MS; $50 \%$ de MS distribuée) et d'aliments concentrés, ont été distribuées selon le schéma du carré latin $(4 \times 4)$. Elles comprenaient l'aliment concentré témoin $(87,4 \%$ de MS; $28,9 \%$ de matières azotées totales; $5,9 \%$ de matières grasses) ou le même concentré supplémenté par $5 \%$ d'un mélange de suif + saindoux $(50 / 50)$, d'huile de soja ou de Mégalac (expérience 1 ), ou $5 \%$ de saindoux, $25 \%$ de graines de soja toastées ou $25 \%$ de tourteau de coprah $+20 \%$ de tourteau de palmiste (expérience 2). La moitié du concentré était distribuée à la traite de $7 \mathrm{~h} 00$ et l'autre moitié à celle de $17 \mathrm{~h} 00$, respectivement; après que le concentré était consommé, l'ensilage de maïs était offert également en 2 repas égaux. Les rations étaient calculées de façon à couvrir les besoins d'entretien et de production laitière. Les enregistrements du comportement alimentaire et mérycique ont été réalisés pendant 5 j pour chaque ration, au moyen d'un système d'acquisition de données (Beauchemin et al, 1989). Chaque période de mesure était précédée d'une période d'adaptation de 3 sem.

Résultats et discussion - Les résultats relatifs au comportement alimentaire et mérycique des animaux sont consignés au tableau I. La nature des suppléments lipidiques n'a quasiment pas affecté la durée unitaire d'ingestion ( $\mathrm{min} / \mathrm{kg} \mathrm{MS}$ ) de l'aliment concentré par rapport au témoin, excepté pour le tourteau de coprah + palmiste, qui a notablement augmenté cette durée. Les résultats correspondants pour l'ensilage de maïs étaient variables, et les différences non significatives. La durée unitaire de rumination (min/ $\mathrm{kg}$ MS totale ingérée) a également été influencée par les différents suppléments lipidiques: elle a été plus élevée avec l'huile de soja et plus faible avec les graisses animales et le Mégalac; une tendance très nette vers l'augmentation a également été notée avec le tourteau de coprah + palmiste. Ce dernier supplément a d'ailleurs notable- 
ment augmenté la durée uniaire de mastication totale (ingestion + rumination).

En conclusion, l'introduction de sources lipidiques dans les aliments concentrés affecte très peu la durée unitaire de leur ingestion, excepté le tourteau de coprah + palmiste qui augmente cette durée. Le rôle des acides gras de longueur moyenne dans cette diminution d'appétibilité devra être vérifié dans des essais ultérieurs. L'apport important d'acides gras insaturés par l'huile de soja affecte surtout la rumination, ce qui ne se reproduit pas lorsque ces acides gras sont moins accessibles aux micro-organismes du rumen, comme c'est le cas avec les graines de soja toastées. II est possible que l'huile de soja entrave la digestion microbienne dans le rumen et que ce phénomène soit compensé par une augmentation de l'activité de mastication durant la rumination.

Remerciements - Travail subventionné par I'IRSIA, rue de Crayer 6, 1050 Bruxelles, Belgique.

Anderson MJ, Lambs RC, Walter JL (1979) $J$ Dairy Sci 63 (suppl 1), 154

Beauchemin KA, Zelin S, Genner D, BuchananSmith JG (1989) J Dairy Sci 72, 2746-2759

Doreau M (1984) Journées du CAAA, INA, Paris-Grignon

Tableau I. Composition des aliments concentrés, production laitière, matière sèche ingérée et comportement alimentaire et mérycique (min $/ \mathrm{kg} \mathrm{MS}$ ) chez les vaches laitières.

\begin{tabular}{|c|c|c|c|c|c|c|c|c|}
\hline & \multicolumn{4}{|c|}{ Expérience 1} & \multicolumn{4}{|c|}{ Expérience 2} \\
\hline & Témoin & $\begin{array}{l}\text { Graisses } \\
\text { de } \\
\text { estruction }\end{array}$ & $\begin{array}{l}\text { Huile } \\
\text { de soja }\end{array}$ & Mégalac & Témoin & Saindoux & $\begin{array}{r}\text { Graines } \\
\text { de soja } \\
\text { toastées }\end{array}$ & $\begin{array}{c}\text { Tourteau de } \\
\text { coprah- } \\
\text { palmiste }\end{array}$ \\
\hline \multicolumn{9}{|l|}{$\begin{array}{l}\text { Composition des } \\
\text { concentrés (\% de MS) }\end{array}$} \\
\hline Matière azotées tot & 28,9 & 27,2 & 27,4 & 27,4 & 26,05 & 25,85 & 29,43 & 23,42 \\
\hline Extrait éthéré & 5,9 & 11,1 & 10,5 & 9,0 & 4,82 & 8,73 & 9,00 & 7,52 \\
\hline Production laitière $(\mathrm{kg} / \mathrm{j})$ & 19,0 & 18,8 & 18,5 & 19,7 & 21,5 & 22,5 & 24,4 & 21,0 \\
\hline $\begin{array}{l}\text { Matière sèche ingérée } \\
(\mathrm{kg} / \mathrm{j})\end{array}$ & 13,7 & 13,2 & 13,2 & 13,2 & 15,7 & 15,9 & 16,9 & 15,6 \\
\hline \multicolumn{9}{|l|}{ Durées de mastication } \\
\hline Ingestion totale & 10,8 & 10,1 & 9,8 & 9,7 & 8,9 & 8,5 & 8,3 & 10,2 \\
\hline Concentré & 5,6 & 5,5 & 5,4 & 5,3 & $4,2^{\mathrm{a}}$ & $4,2^{b}$ & 4,5 & $6,1^{\mathrm{ab}}$ \\
\hline Ensilage & 16,0 & 14,8 & 14,2 & 13,4 & 13,3 & 12,7 & 12,0 & 14,1 \\
\hline Rumination & 28,7 & $26,8^{a}$ & $30,1^{\text {ad }}$ & $25,3^{d}$ & 19,8 & 18,1 & 17,0 & 22,8 \\
\hline Mastication totale & $39,5^{d}$ & 36,9 & $39,8^{d}$ & 34,9 ad & 28,7 & $26,7^{a}$ & 25,3 & $33,0^{\mathrm{ab}}$ \\
\hline
\end{tabular}

Dans une méme ligne et un même essai, les moyennes munies d'un indice commun sont significativement différentes (a,b,c: $P<0,10 ; \mathrm{d}: P<0,05)$. 